\title{
Experimental demonstration of crowd synchrony and first-order transition with lasers
}

\author{
Simon Mahler $\odot,{ }^{*}$ Asher A. Friesem $\odot$, and Nir Davidson \\ Department of Physics of Complex Systems, Weizmann Institute of Science, Rehovot 761001, Israel
}

(Received 22 July 2020; accepted 27 October 2020; published 12 November 2020)

\begin{abstract}
Synchronization of different and independent oscillators that interact with each other via a common intermediate is ubiquitous in many fields. Here, we experimentally demonstrate the effect of crowd synchrony, analogous to that of the Millennium Bridge, by resorting to coupled lasers. When the number of lasers is below a critical number, there is no synchronization, but after reaching the critical number, the lasers instantaneously synchronize. We show that the synchronization of the lasers as a function of their number follows a first-order transition, and that our experimental results are in good agreement with those predicted by theoretical models.
\end{abstract}

DOI: 10.1103/PhysRevResearch.2.043220

\section{INTRODUCTION}

Synchronization of independent dynamical elements via an intermediate medium plays an important role in biology, chemistry, engineering, and physics. It was investigated in cell and molecular biology [1-5], chemical oscillators [6-8], bridge engineering [9-14], humans walking [13,15], optics and lasers [16,17], collective behavior of species and microorganisms [18], social systems [19], clocks [20,21], chaotic oscillators [22-24], and networks [22,23,25]. In most of the cases, the synchronization depends on the number of elements where a critical number determines the onset of the synchronization. Representative examples where such an onset occurs are in quorum sensing and crowd synchrony. In quorum sensing, which was initially observed when studying collective behaviors and interactions of cells $[1,3,26]$, the dynamics and oscillations of the elements only occur above a critical density with a first- or second-order transition to synchronization $[1,6,16,24]$.

Crowd synchrony was first investigated and modeled on the pedestrian Millennium Bridge in London, which was closed shortly after its opening day due to uncontrolled strong lateral oscillations. The pedestrians, each walking at different pace and speed, caused small lateral oscillations to the bridge, which in turn caused the pedestrians to sway in step in order to retain balance, dramatically amplifying the oscillations of the bridge and synchronizing the pedestrians $[9,11,14,15]$. The effect was modeled as crowd synchronization [Fig. 1(a)], where the lateral oscillations of the bridge were attributed to a combination of oscillations and synchronization that critically depends on the number of pedestrians $[10,11]$. Subsequently, the crowd synchrony model was applied to different systems including chaotic oscillators [24], networks [17,23,25], and chemical oscillators [6].

\footnotetext{
*sim.mahler@gmail.com

Published by the American Physical Society under the terms of the Creative Commons Attribution 4.0 International license. Further distribution of this work must maintain attribution to the author(s) and the published article's title, journal citation, and DOI.
}

Crowd synchrony was also theoretically predicted with coupled lasers, where coupling between $M$ independent (star) lasers (analogous to pedestrians) is mediated by a central (hub) laser (analogous to the bridge) operating below lasing threshold [Fig. 1(b)] [16]. When $M$ is above a critical number $M_{c}$, the lasers synchronize with a first-order transition where $M_{c}$ depends on the coupling strength between the star lasers and the hub laser, on the frequency distribution of the lasers, and on the pumping strength $[11,16,17]$.

Phase synchronization of independent lasers has been extensively investigated experimentally during the last decade. These investigations include laser beam combining to obtain high brightness outputs for high-power laser applications, simulating spin systems [27-29], finding the ground-state solution of complex landscapes [30-32], observing dissipative topological defects [33], solving the phase retrieval problem [34], and more [35]. Recently, phase synchronization of laser arrays was achieved with nonlinear (spatiotemporal) coupling by means of a saturable absorber, demonstrating improvement in finding a ground-state solution in comparison with linear coupling [30].

While these experimental investigations yielded many exciting results on synchronization, they did not provide information on the nature of the transition to synchronization. Numerical investigations showed that the onset of the synchronization can depend on the number of lasers [36-39]. Specifically, for a large number of coupled lasers, the transition to synchronization was predicted to change from second order to first order if accompanied by a lasing transition [40], but such prediction was not verified experimentally.

In this work, we present an experimental demonstration of crowd synchrony with coupled lasers. It is achieved with two coupled degenerate cavity lasers (DCLs) [41]. The first DCL forms a controllable number of independent (star) lasers with nearly uniform intensities but different phases and frequencies. The second (hub) DCL, operating below lasing threshold, can phase synchronize the independent star lasers when their number increases. We observed a first-order transition to synchronization as the number of star lasers crosses a critical number $M_{c}$ that depends on the coupling strength $K$ between the two DCLs and follows a power law with 


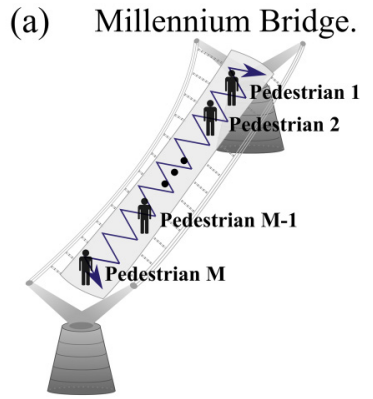

(b) Lasers.

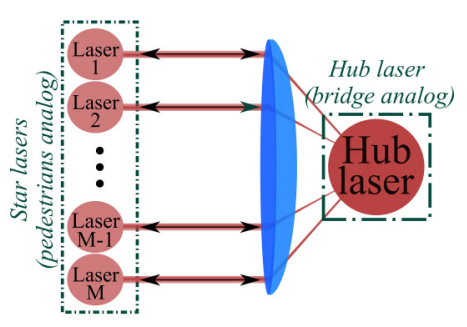

(c) Degenerate cavity lasers arrangement.

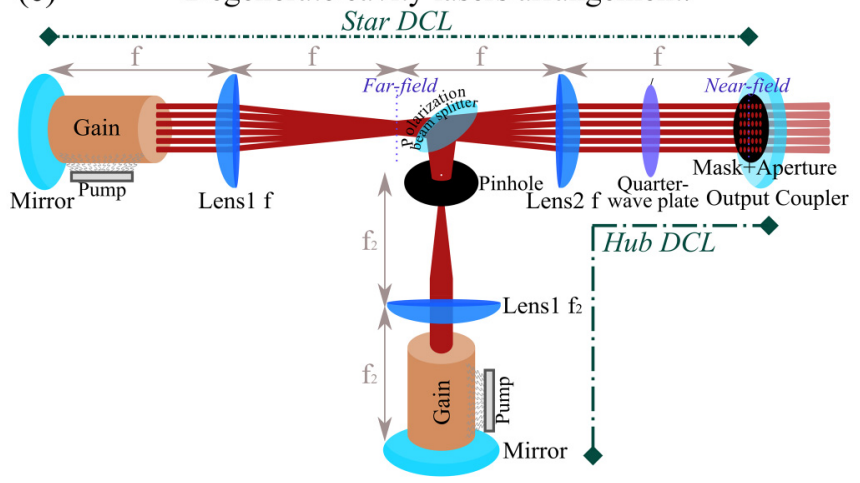

FIG. 1. Crowd synchronization. (a) Millennium Bridge, which is immobile with few pedestrians and laterally oscillating with many pedestrians [9,10]. (b) Model for crowd synchrony with (star) lasers coupled via a (hub) laser mediate, where there is no phase synchronization with few lasers and phase synchronization with many lasers [16]. (c) Arrangement for experimentally demonstrating crowd synchrony composed of two self-imaging degenerate cavity lasers (DCLs) coupled with adjustable coupling strength $K$ via polarization elements. The multimode star DCL serves to form independent (uncoupled) lasers and the single-mode hub DCL, operating below its lasing threshold, mediates all-to-all coupling between them. A variable size near-field aperture and an amplitude mask adjacent to the output coupler control the number of star lasers.

a scaling exponent $v=-2.4$, in good agreement with the theoretically predicted one $v_{t h}=-2.2$ [16]. This scaling law is independent of the array geometry, providing evidence of universality. We also show that the sharp transition to synchronization is related to the onset of the lasing transition of the hub laser, providing the feedback needed for a first-order transition [6].

In the following, Sec. II presents the experimental arrangement and results of crowd synchronization with lasers, where a first-order transition to synchronization is observed. Additional details about the experimental arrangement, numerical simulations, and the characterization of the synchronization are given in Appendices A and B. Section III presents a scaling exponent associated to the transition and provides experimental and numerical evidence of universality. Additional numerical simulations are presented in Appendix C. Section IV discusses the synchronization when the hub laser is set above lasing threshold. Section $\mathrm{V}$ describes the lasing of the hub laser during the synchronization of the lasers. Finally, concluding remarks are given in Sec. VI.

\section{EXPERIMENTAL ARRANGEMENT AND RESULTS}

Our experimental arrangement for investigating crowd synchrony with coupled lasers is schematically presented in Fig. 1(c). It includes two DCLs coupled with a tunable coupling strength $K$ [42]. One DCL (horizontal orientation) has a high number of transverse modes and forms at the near-field plane an array of independent and uncoupled (star) lasers with any desired geometry $[43,44]$. A variable size aperture controls the number of star lasers. A second (hub) DCL with a single transverse mode mediates positive and long-range coupling between the star lasers [43]. The intensity distributions at the near-field plane of the star lasers array and at its far-field plane (corresponding to its coherence function) are both imaged to and detected by a CCD camera. The star lasers operate above lasing threshold and the hub laser below lasing threshold. More details are given in Appendix A.

Using the arrangement of Fig. 1(c), we performed extensive experiments and simulations with square and with random arrays of lasers to demonstrate and study crowd synchronization. The results are presented in Figs. 2-4. Figures 2(a) and 2(b) show representative experimental and simulated near-field and far-field intensity distributions for $M=41$ and 45 lasers, respectively. For $M=41$ lasers, the far-field intensity distribution is broadly distributed, indicating that the lasers are uncoupled [44]. For $M=45$ lasers, the far-field intensity distribution contains a sharp central peak, indicating stable in phase locking (synchronization) between the lasers [43]. We determined that $80 \%$ of the $M=45$ lasers were phase synchronized (see Appendix B 4). Numerical simulations performed by a combined algorithm [45] that includes the Fox-Li [46] and the Gerchberg-Saxton [47] algorithms are in good agreement with the experimental data (see Appendix A).

Both the experimental and simulation results indicate that synchronization critically depends on the number of star lasers. Below a critical number $\left(M_{c}=43\right.$ lasers here $)$, the star lasers remain uncoupled whereby their energy is not sufficient to pump and enforce lasing in the hub laser. Such behavior is analogous to that occurring in the Millennium Bridge where below a critical number of pedestrians there are no lateral oscillations. Above $M_{c}$, the star lasers phase synchronize, thereby providing sufficiently strong coherent signal to drive the hub laser above its lasing transition, which couples the star lasers and synchronize them.

To quantify the results, we repeated these experiments for many different numbers of star lasers and characterized the synchronization using the (normalized) power of the coherent DC peak in the far-field plane (Fourier transform) of the lasers, similarly to the total coherent intensity used in [16]. The coherent peak ratio of the phase-locked lasers was calculated by using the cross section along the center of the far-field intensity distributions (as in Fig. 2, fourth column). First, the background intensities in the cross section were fitted with a Gaussian. Then, the integrated intensity above the Gaussian fit was divided by the total integrated intensity (see Appendix B for more details). A high coherent peak ratio indicates that most of the lasers are synchronized in phase.

Figure 2(c) shows the coherent peak ratio as a function of the number of star lasers. As evident, a sharp first-order 

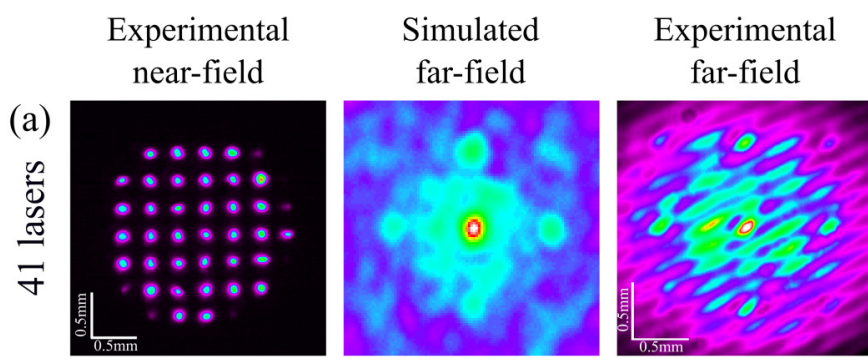
Experimental far-field cross section
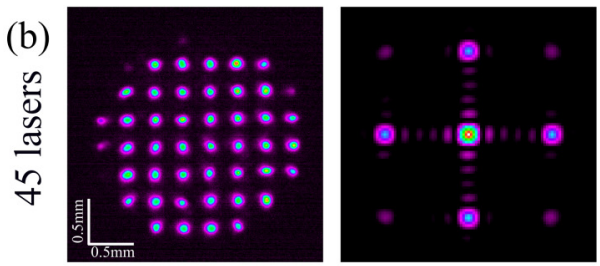
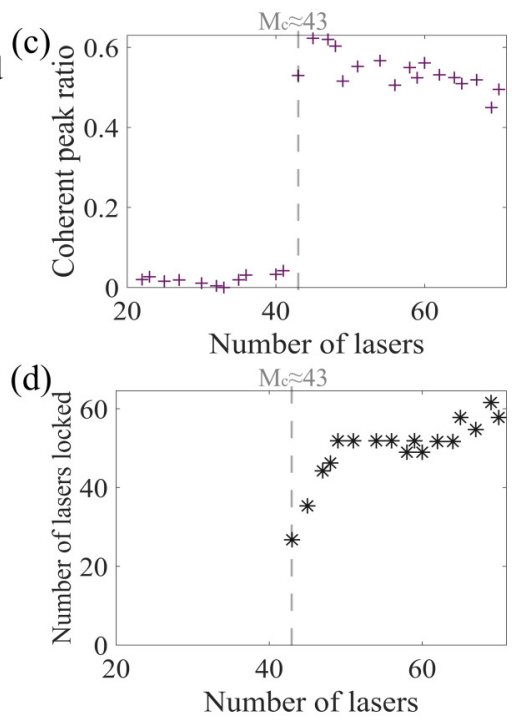

FIG. 2. Crowd synchronization in a square array of lasers. (a), (b) Experimental and simulated near-field and far-field intensity distributions with corresponding cross sections along the far-field center for square array of star lasers with coupling $K \approx 0.37$. (a) $M=41$ lasers where the broad far-field intensity distribution indicates no phase synchronization. (b) $M=45$ lasers, where the sharp far-field peaks indicate phase synchronization. (c) Coherent peak ratio as a function of the number of star lasers revealing a sharp first-order transition to synchronization, at a critical number $M_{c}=43$. (d) Number of phase-synchronized lasers as a function of the number of star lasers.

transition occurs at $M_{c}=43$ between low and high plateau values of the coherent peak ratio. The high plateau value above $M_{c}=43$ corresponds to a sharp high-intensity peak ratio, as would occur for in-phase synchronized lasers. Alternative synchronization criteria yielded similar results (see Appendix B). Figure 2(d) shows the number of phase-locked (synchronized) lasers (see Appendix B) as a function of the number of lasers. At $M>M_{c}$, the number of phase-locked lasers sharply increases and continues to increase, indicating that most of the lasers are synchronized.
Next, we repeated all the experiments and measurements for a random array of lasers where each laser was randomly positioned in space with fixed distance $a$ between nearest neighbors. Figures 3(a) and 3(b) show representative experimental and simulated near-field and far-field intensity distributions for $M=25$ and 30 lasers, respectively. For $M=$ 25 lasers, the far-field intensity distribution is broadly distributed, indicating that the lasers are uncoupled. For $M=30$ lasers, the far-field intensity distribution contains a sharp central peak, indicating that the lasers are phase synchronized.
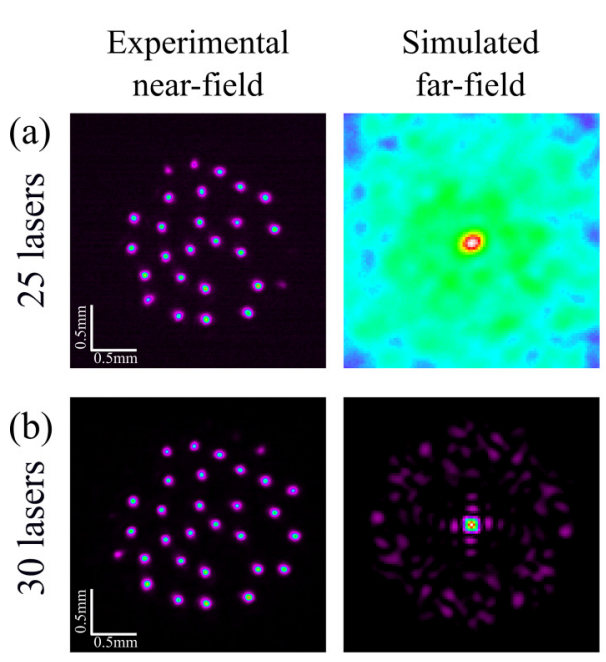
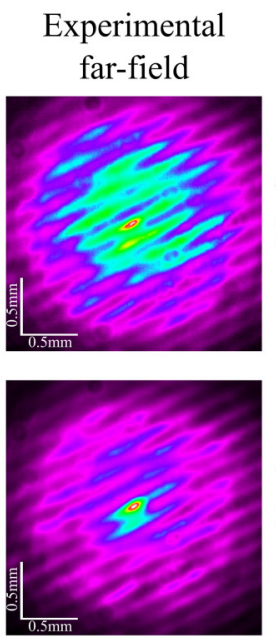
Experimental far-field cross section
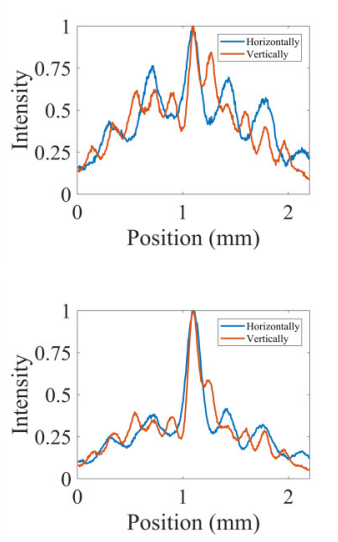

(d)
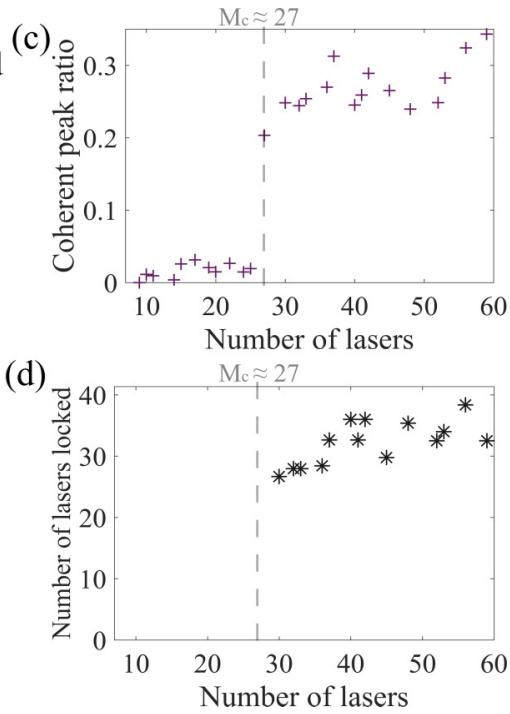

FIG. 3. Crowd synchronization in a random array of lasers. (a), (b) Experimental and simulated near-field and far-field intensity distributions with corresponding cross-section intensities along the far-field center for random array of star lasers with coupling $K \approx 0.46$. (a) $M=25$ lasers where the broad far-field intensity distribution indicates little or no phase synchronization and (b) $M=30$ lasers where the sharp far-field intensity peak indicates phase synchronization. (c) Coherent peak ratio as a function of the number of star lasers revealing a sharp transition to synchronization, at a critical number $M_{c}=27$. (d) Number of phase-synchronized lasers as a function of the number of star lasers. 
Figure 3(c) shows the coherent peak ratio of the farfield intensity distributions as a function of the number of lasers. As for the square array, a sharp first-order transition to synchronization occurs at a critical number $M_{c}=27$ lasers. Figure 3(d) shows that above $M_{c}$ most of the lasers are phase synchronized. The results for the random laser array are similar to those of the square array, indicating that the geometry of the array does not influence the synchronization of the lasers.

\section{UNIVERSALITY AND SCALING EXPONENT}

We repeated the experiments and measurements for both square and random laser arrays with different coupling strengths $K$ between the star and the hub DCLs, and determined the critical number of lasers $M_{c}$ as a function of $K$ (see Appendix A). The results, presented in Fig. 4(a), indicate that $M_{c}$ does not depend on the array geometry and decreases as the coupling increases.

The results for both array geometries are well fitted by a power law $M_{c} \propto K^{v}$ with a scaling exponent $v=-2.4$, significantly different from the scaling exponent $v_{t h}=-1$ predicted for pedestrians walking on the Millennium Bridge [10]. We attribute this difference to the highly nonlinear lasing transition of the hub laser that plays an important role in synchronizing the star lasers (as opposed to the linear response of the bridge to the force applied by the pedestrians).

Numerical simulations were also performed as detailed in Appendix A. The results, presented in Fig. 4(b) for a square array of lasers when the pump value of the hub laser was set below threshold, are in qualitative agreement with the experimental results. The numerical scaling exponent $v_{\text {num }}=-2.2$ is in agreement with the theoretically predicted one for timedelayed coupled lasers [16]. Additional numerical simulations investigating the dependence of the scaling exponent on the laser parameters were performed in Appendix C.

\section{SYNCHRONIZATION WHEN THE HUB LASER IS SET ABOVE LASING THRESHOLD}

Next, we studied crowd synchrony when the pump value of the hub laser is above lasing threshold. The results are presented in Fig. 5, showing the measured coherent peak ratio
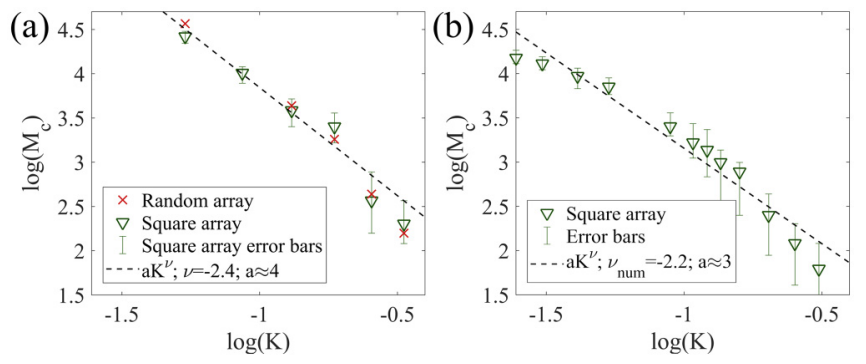

FIG. 4. Universality and scaling exponent in crowd synchronization. Critical number of lasers $M_{c}$ as a function of the coupling strength $K$ between the two DCLs for square and random laser arrays. (a) Experimental and (b) numerical. The critical number of lasers decreases as the coupling increases and is fitted by a power law $M_{c} \propto K^{v}$ (dashed line) with (a) $v=-2.4 \pm 0.35$ and (b) $v_{\text {num }}=$ $-2.2 \pm 0.30$. Error bars for the random array (not shown) are similar to those of the square array.
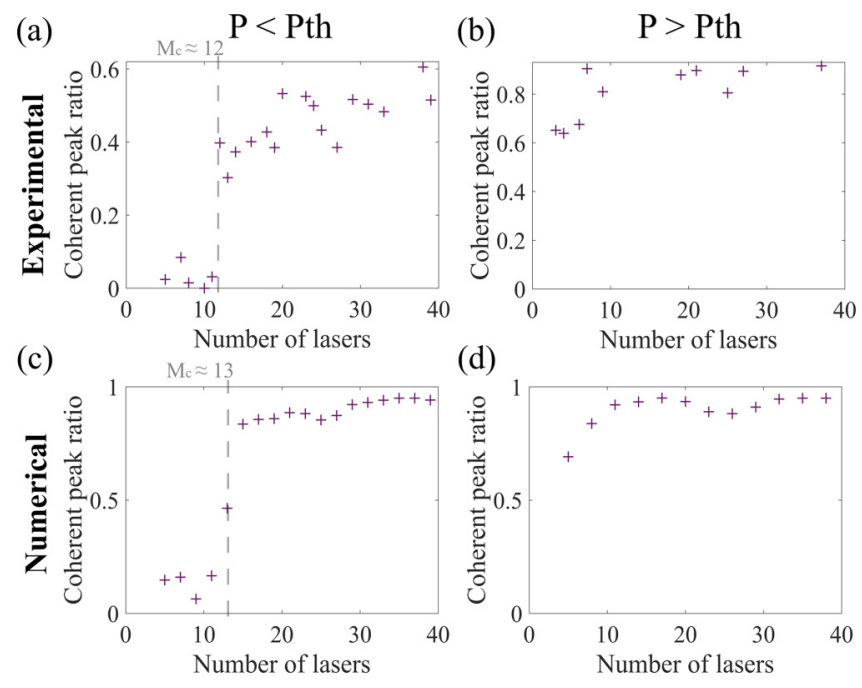

FIG. 5. Synchronization of the lasers for two different pump values of the hub laser. (a), (b) Experimental. (c), (d) Numerical. Below threshold $\left(P<P_{t h}\right)$, a jump in the coherent peak ratio indicates a transition to synchronization at $M_{c}$ star lasers. Above threshold $\left(P>P_{t h}\right)$, the coherent peak ratio varies smoothly with the number of star lasers.

as a function of the number of star lasers for two different pump values. As evident in Fig. 5(a), when the pump value of the hub laser is below lasing threshold, the synchronization of the lasers follows a sharp first-order transition at $M_{c}=12$ star lasers. When the pump value is above lasing threshold [Fig. 5(b)], synchronization occurs even at a small number of star lasers and changes smoothly as the number of star lasers increases. The numerical simulations, presented in Figs. 5(c) and 5(d), are in good agreement with the experimental results.

When the hub laser is set above lasing threshold, synchronization depends smoothly on the number of star lasers [16], in analogy to crowd dynamics on a wobbly bridge [12]. A similar synchronization situation occurs in coupled chemical oscillators [6]. For strong coupling, each oscillator is affected by the other oscillators with a positive feedback that leads to a dynamical instability and a sharp transition to synchronization as the density of oscillators is increased. For weak coupling, the (autocatalyst) concentration of each oscillator is unaffected by the others, leading to a smooth transition to synchronization. Chemical oscillators with fixed concentrations and also lasers with fixed intensities are well described by the Kuramoto model [33] that predicts a second-order transition even with all-to-all couplings. Sharp first-order transition only occurs when the chemical concentrations or laser intensities can vary $[40,48]$.

\section{LASING OF THE HUB LASER WHEN $M<M_{c}$}

Finally, using the experimental arrangement of Fig. 1(c), we studied the relation between crowd synchrony and the transition to lasing of the hub laser. The results are presented in Fig. 6, which shows the far-field intensity distributions of the star lasers and of the combined hub and star lasers, for different numbers of star lasers in a square array. The top row shows the measured far-field intensity distributions of the star 


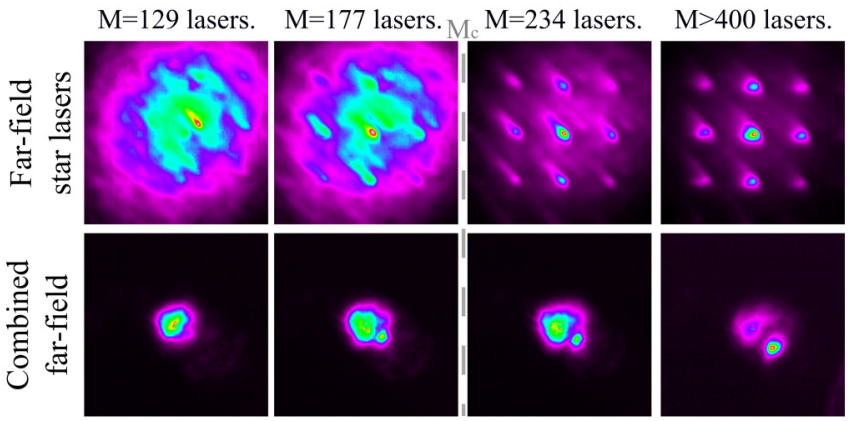

FIG. 6. Lasing of the hub laser when $M<M_{c}$. Top row: far-field intensity distributions of different number of star lasers in a square array, showing a transition to synchronization at about 200 star lasers. Bottom row: combined far-field intensity distributions of the star and hub lasers showing a second peak emerging at $M=177$ star lasers, corresponding to the lasing onset of the hub laser, below the transition to synchronization.

lasers detected through the common output coupler of both lasers in Fig. 1(c). As evident, the results are similar to those in Fig. 2 (but with a weaker coupling), showing a transition to synchronization at about 200 star lasers. Note that these intensity distributions also include some light from the hub laser but of lower intensity compared to the 200 star lasers.

The bottom row shows the measured far-field intensity distributions detected through the back mirror of the hub DCL [mirror 2 in Fig. 1(c)]. They mainly include light from the hub laser and a small fraction from the star lasers (transmitted through the hub laser cavity and its intracavity pinhole). For $M<177$ lasers, the intensity distributions are comprised of a single peak from the star lasers that gradually increases as the number of lasers increases. At $M=177$ star lasers, a second sharp peak appears next to the first one. As the number of star lasers further increases, the intensity of the second peak increases while the intensity of the first peak decreases.

We attribute this second sharp peak to the lasing of the hub laser (that did not fully overlap with the star lasers peak due to a small misalignment between the star and hub DCLs). The lasing onset of the hub laser thus appears around $M=177$ lasers, below the transition to synchronization, in analogy to the dynamics of feedback-stabilized walking bipeds [15] and to a double transition to synchronization [49].

\section{CONCLUSIONS}

To conclude, we have shown experimentally that an array of star lasers coupled to a common hub laser can synchronize, similarly to crowd synchrony of pedestrians on the Millennium Bridge. The synchronization follows a first-order transition as the number of lasers crosses a critical number. The critical number of lasers obeys a power law as a function of the coupling strength with a scaling exponent $v=-2.4$, independently of the array geometry but different from that of the Millennium Bridge, reflecting the important role of the highly nonlinear lasing transition in synchronization. The transition to synchronization of coupled lasers by the lasing transition has attracted a lot of attention for a variety of research activities, simulating spin systems [28,30,34], solving computational problems [27-29,34], studying topological dynamics [33], and coherent beam combining [43,44]. Crowd synchrony and its associated sharp and universal transitions can advance our understanding in these research areas and also open new ones. Our investigations were centered on crowd synchrony, but the experimental arrangement could be adapted to deal with more general synchronization or quorum sensing situations.

\section{ACKNOWLEDGMENTS}

The authors wish to acknowledge the Israel Science Foundation, the Israel Ministry of Science, Technology and Space, and the Israeli Planning and Budgeting Committee Fellowship Program for their support and thank H. Nakav, R. Chriki, and C. Tradonsky for valuable help.

\section{APPENDIX A: MATERIALS AND METHODS}

\section{Experimental arrangement}

The experimental arrangement schematically presented in Fig. 1(c) includes two self-imaging DCLs coupled together by means of a quarter-wave plate and a polarization beam splitter that control the coupling strength $K$ between them. The first DCL (horizontal orientation) has a high number of transverse modes and forms an array of independent and uncoupled lasers $[43,44]$, corresponding to the star lasers. We estimated the level of frequency detuning between the star lasers to be few $\mathrm{MHz}$ [50]. The second DCL has a single transverse mode and serves to couple the lasers positively with long range [43], corresponding to the hub laser. Each DCL is comprised of a back mirror of high $>95 \%$ reflectivity, a $\mathrm{Nd}$ :YAG gain medium rod of $0.50-\mathrm{cm}$ diameter and $10.9-\mathrm{cm}$ length (operating wavelength $\lambda=1064 \mathrm{~nm}$ ) that is optically pumped by a quasi-CW $100-\mu$ s duration pulsed flash lamp operating at $1 \mathrm{~Hz}$ to minimize thermal lensing, a spherical lens Lens1 of focal length $f=20 \mathrm{~cm}$ for the star lasers or $f_{2}=15 \mathrm{~cm}$ for the hub laser at focal distance from the back mirror, a polarization beam splitter that combines the laser fields of the star and hub lasers, forming a common laser field, a second spherical lens Lens 2 of focal length $f=20 \mathrm{~cm}$ at focal distance $f$ from the focal plane of Lens 1 and a common mirror to both laser fields of $95 \%$ reflectivity at the front focal plane of Lens 2 that serves as an output coupler.

A mask of a square (or random) array of holes with diameter $200 \mu \mathrm{m}$ separated by a period $a=300 \mu \mathrm{m}$, adjacent to the output coupler (denoted as the near field) is used to form an array of star lasers. A variable size aperture is placed next to the mask of holes to control the number of star lasers. Lenses Lens 1 and Lens2 form a telescope configuration yielding a self-imaging condition, ensuring that each hole in the mask forms an independent laser. At the focal plane midway between Lens1 and Lens2 of the hub laser (far-field Fourier plane), a pinhole of size below the diffraction limit couples the lasers positively with long-range coupling. The near-field and far-field intensity distributions are both imaged to and detected by a CCD camera. The star lasers operate above lasing threshold and the hub laser below lasing threshold. All the far-field intensity distributions that support the plots within this paper were averaged over five realizations. 


\section{Numerical simulation}

For the simulation of the far-field intensity distributions in Figs. 2 and 3, we resorted to a combined algorithm that includes the Fox-Li and the Gerchberg-Saxton algorithms. It is an iterative algorithm that numerically mimics the propagation in space of the laser field in the DCL where an iteration in the algorithm numerically mimics a roundtrip of the laser field in the DCL. For that, a two-dimensional matrix $E_{i j}$ representing the spatial distribution of the laser field is initialized with uniform intensities and random phases. During each iteration, the initial laser field $E_{i j}$ is multiplied by a saturable gain function $G_{i j}$, then is Fourier transformed (first far field $\mathrm{FF} 1_{i j}$ ), then is inversely Fourier transformed (near field $\mathrm{NF}_{i j}$ ), then is Fourier transformed again (second far field $\mathrm{FF} 2_{i j}$ ) and finally is inversely Fourier transformed (laser field $E_{i j}$ ). The resulting laser field $E_{i j}$ is kept and used for the next iteration.

The saturable gain function is $G_{i j}=\frac{G_{0}}{1+\left|E_{i j}\right|^{2} / I_{\text {sat }}}$, where $G_{0}$ is the constant pump value and $I_{s a t}$ the saturation intensity. Components inserted in the near-field or far-field planes of the DCL, such as mask of holes or pinhole, can be numerically mimicked in the algorithm by multiplying the near-field $\mathrm{NF}_{i j}$ or far-field $\mathrm{FF}_{i j}$ matrices by the Pinhole $i j$ or Mask $_{i j}$ matrices.

The experimental arrangement of Fig. 1(c) was simulated by using two different laser fields $E_{i j}^{\text {star }}$ and $E_{i j}^{\text {hub }}$ each one representing the star and hub DCLs, respectively. For the star DCL, the laser field $E_{i j}^{\text {star }}$ is multiplied by $G_{i j}^{\text {star }}$, Fourier transformed, inversely Fourier transformed, multiplied by Mask $_{i j}$, phase detuned, Fourier transformed, added to the laser field from the hub laser with coupling $1-K$, and finally inversely Fourier transformed. Phase detuning was performed by adding random phase noise to the field of the star lasers with $0.7 \%$ relative intensity magnitude. We checked other intensity magnitudes of noise $I_{\xi}$ of the random phase field and obtained similar results (see Appendix C).

For the hub DCL, the laser field $E_{i j}^{\text {hub }}$ is multiplied by $G_{i j}^{\text {hub }}$, Fourier transformed, multiplied by Pinhole $_{i j}$, inversely Fourier transformed, multiplied by Mask $_{i j}$, Fourier transformed, added to the laser field from the star laser with coupling $K$, and finally inversely Fourier transformed. The pump value $G_{0}^{\text {star }}$ of the star DCL was set above lasing threshold and the pump value $G_{0}^{\text {hub }}$ of the hub DCL was set below lasing threshold. The mask of holes was either a square or random array geometry and the pinhole size was set below the diffraction limit of the DCL. The simulations were performed with 150 iterations and were averaged over different realizations to account for the different longitudinal modes of the DCL [45].

\section{Determining the critical number of lasers $M_{c}$}

The critical number of lasers $M_{c}$ was determined by fitting the coherent peak ratio (CPR) by the function CPR = $A+\frac{B}{1+\exp \left[-k\left(M-M_{c}\right)\right]}$, where $M$ is the number of lasers, $A$ and $B$ are the CPR values of the low and high plateaus around the transition, $k$ is a constant that denotes the sharpness of the transition, and $M_{c}$ is the critical number of lasers. From the fitting, the errors on the critical number of lasers $M_{c}$ were determined according to two-sigma (95\%) confidence.

\section{Calculating the coupling $K$}

The coupling $K$ between the star and hub DCLs corresponds to the fraction of light transferred from the star lasers to the hub laser by the polarization beam splitter. This fraction is varied by rotating the quarter-wave plate in Fig. 1(c). Using Malus's law, the coupling can be calculated as $K=\sin ^{2}(4 \theta)$, where $\theta$ is the angle between the horizontal axis and the fast axis of the quarter-wave plate.

\section{APPENDIX B: CHARACTERIZING THE SYNCHRONIZATION}

We characterized the synchronization of the lasers by calculating the normalized phase-locking (coherent) intensity of the lasers from the far-field intensity distributions, similarly to the total coherent intensity calculated in [16]. From the farfield (coherence) intensity distributions, we measured the DC peak normalized intensity at the center by using three different criteria: the coherent peak ratio, the inverse participation ratio (IPR), and ratio of intensities (RI). We also calculated the number of phase-locked lasers.

\section{Coherent peak ratio}

The coherent peak ratio was calculated by using the cross sections along the center of the far-field intensity distributions. Two representative cross sections are shown in Fig. 7, one with a low coherent peak ratio and the other with a high coherent peak ratio. To determine the coherent peak ratio, first the background intensities in the cross sections were fitted with a Gaussian, and then the integrated intensity above the Gaussian fit (peak signal, i.e., the blue area in Fig. 7) was divided by the total integrated intensity (orange area in Fig. 7). A high coherent peak ratio indicates that there is a high intensity central peak in the cross section and that most of the lasers are in-phase synchronized. The coherent peak ratio depends on the Gaussian fitting. So, we checked the quality of the Gaussian fitting on all the cross sections along the center in the far-field intensity distributions.

\section{Inverse participation ratio (IPR)}

The inverse participation ratio (IPR) is widely used for measuring the localization of a state, mostly in real space.
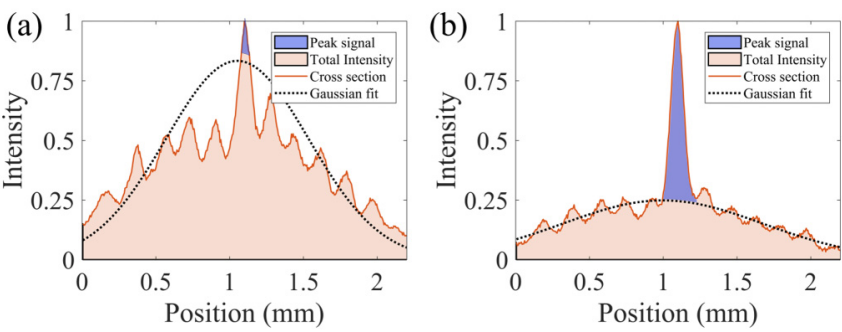

FIG. 7. Coherent peak ratio determination. Cross sections along the center of the far-field intensity distribution for a random array of lasers, (a) for a low coherent peak ratio and (b) for a high coherent peak ratio. The peak and background cross-section intensities are both fitted with a Gaussian and then the integrated intensity of the peak signal is divided by the total integrated intensity. 
When the IPR is applied in the Fourier space, it can characterize the onset of phase synchronization of oscillators [51]. We used the far-field intensity distributions to calculate the IPR according to

$$
\mathrm{IPR}=\frac{\left[\sum_{i} I_{i}\right]^{2}}{\sum_{i} I_{i}^{2}}
$$

where $I_{i}$ is the intensity of the $i$ th pixel in the far field and the sums occur over all the pixels. The IPR indicates whether sharp peaks occur in the far-field intensity distribution and is therefore a quantitative measure of synchronization. Unlike the coherent peak ratio, the IPR does not depend on the location of the peaks and can thus measure synchronization for different phase-locking states (e.g., in phase and out of phase).

Low values of IPR indicate sharp peaks in the far-field intensity distributions, corresponding to phase synchronization of the star lasers. The IPR strongly depends on the background intensities, so in order to obtain IPRs that clearly identify sharp peaks, we reduced the effect of background intensities. This was done by setting the intensities in the far-field intensity distribution, which are below $10 \%$ and $30 \%$ of the maximal intensity to zero for square and random arrays, respectively.

\section{Ratio of intensities}

We defined the ratio of intensities as the average background intensity $\left\langle I\left(x_{i}\right)\right\rangle$ over the central peak intensity $I_{M}$ in the cross section along the center of the far-field intensity distribution. Two typical cross-section intensity distributions are presented in Fig. 8, one for a square laser array and the other for a random laser array. The position $x(i)$ corresponds to the positions between the tail of the central peak and the tail of the adjacent peak in the distribution of the square laser array. We assume that the positions are the same for the random laser array (since $D=\lambda f / a$ is constant, see Appendix B 4). A low ratio of intensities indicates that the central peak is much higher than the background intensities.

The synchronization characterization with the ratio of intensities has the advantage that it does not depend on any free parameter, whereby it is directly determined from the cross sections. It also accurately provides the background intensities level. However, it should be noted that such characterization is qualitative and cannot be directly related to the synchronization of the lasers.

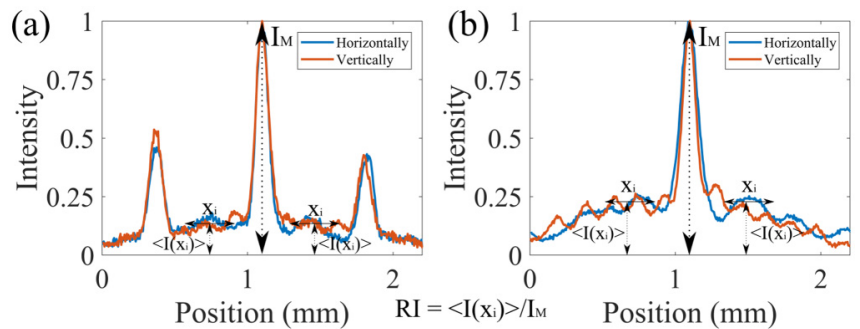

FIG. 8. Ratio of intensities (RI) determination. Two cross sections along the center of the far-field intensity distribution and relevant parameters for (a) a square and (b) a random array of lasers. $\mathrm{RI}=\left\langle I\left(x_{i}\right)\right\rangle / I_{M}$ where $\left\langle I\left(x_{i}\right)\right\rangle$ is the average background intensity and $I_{M}$ the central peak intensity.

\section{Number of phase-locked lasers}

A different method to characterize the synchronization is to calculate the number of phase-locked lasers. We set the number of phase-locked lasers as $G=g_{h} \times g_{v}$ where $g_{h}$ and $g_{v}$ are the number of phase-locked lasers horizontally or vertically with $g=D /$ FWHM where $D$ is the distance between adjacent peaks in the far-field intensity distribution and FWHM is the full width at half-maximum of the central peak [e.g., Fig. 2(b)].

To see this, we note that $D=\lambda f / a$ and $\mathrm{FWHM}=\lambda f / A$, where $\lambda$ is the laser light wavelength, $f$ the focal length of Lens2, $a$ the period of the array of lasers, and $A$ their coherence length. Then, $g=A / a$. In our experiments $\lambda, a$, and $f$ were fixed parameters, so the distance $D$ was the same for all the measurements. Only the FWHM varied.

When the far-field background intensities are high, it is difficult to unambiguously identify the central peak and $G$ fails to accurately determine the number of phase-locked lasers because it is difficult to distinguish the central peak from the background intensities. To overcome this difficulty, we used the ratio of intensities to determine the background intensities level (see Appendix B 3). When the background intensity level was above $40 \%$ of the maximal intensity, i.e., $\mathrm{RI}>\mathrm{RI}_{c}=0.4$, $G$ was set to zero. The value $\mathrm{RI}_{c}=0.4$ was arbitrarily chosen below (and near) 0.5 since the FWHM is calculated at 50\% of the maximal intensity of the central peak. Accordingly, the number of phase-locked lasers depends on the ratio of intensities for identifying uncoupled lasers but is a good and accurate synchronization indicator when the majority of the lasers are coupled.

\section{Comparison of synchronization criteria}

Figure 9 presents the results of all synchronization criteria: (a) coherent peak ratio, (b) IPR, (c) ratio of intensities, and (d) number of phase-locked lasers for the square array of lasers.
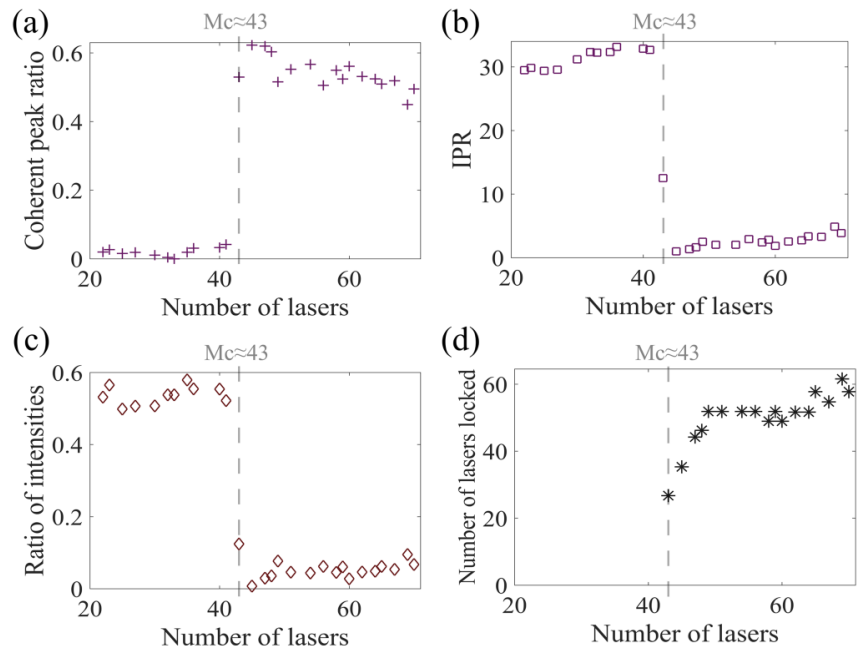

FIG. 9. Crowd synchronization characterization for a square array of lasers with $K \approx 0.37$. (a) Coherent peak ratio, (b) inverse participation ratio (IPR), (c) ratio of intensities as a function of the number of lasers, and (d) number of phase-locked lasers. All follow a sharp first-order transition as a function of the number of lasers, where the critical number of lasers is $M_{c}=43$. 

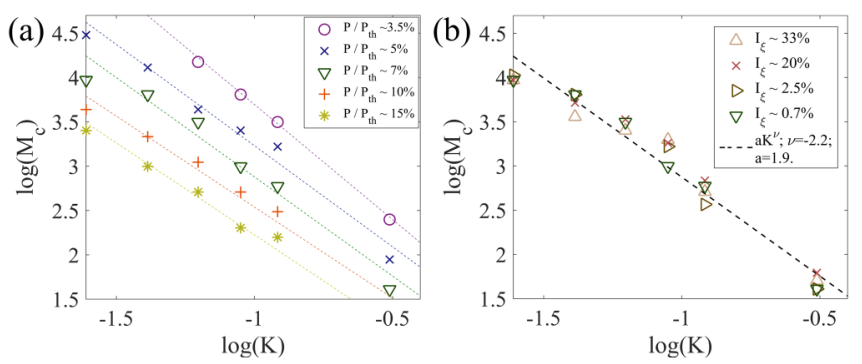

FIG. 10. Universality in numerical simulation, showing critical number of lasers $M_{c}$ as a function of the coupling strength $K$. (a) Different pump ratios $P / P_{t h}$ of the hub laser ( $P_{t h}$ the threshold value). (b) Different intensity magnitudes of the random phase field $I_{\xi}$. The dashed lines denote power-law $\left(M_{c}=a K^{v}\right)$ fit. As evident, all the power-law fits follow the same slope, indicating a similar scaling exponent $v$.

Figures 9(a) and 9(d) are identical to Figs. 2(c) and 2(d). As evident, there is a good agreement between all these synchronization criteria, where there is a sharp transition to synchronization at $M \sim M_{c}$. Similar results were also obtained with all synchronization criteria for the random array of lasers.

\section{APPENDIX C: UNIVERSALITY IN NUMERICAL SIMULATION}

In Sec. III, a scaling exponent $v$ associated to the transition to synchronization was experimentally and numerically investigated [Figs. 4(a) and 4(b)]. Experimental evidence of universality was found by repeating the experiments with a different array of lasers in Fig. 4(a).

We numerically investigated the dependence of the scaling exponent on the laser parameters such as the pump value of the hub laser (active media) and the intensity magnitude of the random phase field (passive media, see Appendix A 2 for more details). The results are presented in Fig. 10. Figure 10(a) shows the $\log$ of the critical number of lasers $M_{c}$ as a function of the log of the coupling strength $K$, for different pump ratios $P / P_{t h}$ of the hub laser where $P_{t h}$ is the pump threshold value. For each pump ratio, the critical number of lasers decreases as the coupling increases and is fitted (dashed lines) with a power law $M_{c}=a K^{\nu}$. For all pump ratios, the power-law fit follows a similar slope, indicating a similar scaling exponent $v_{P}$ with a different amplitude $a$ between them. The scaling exponent $v_{P}$, calculated from the power-law fit, varied in the range from -2.5 to -2.1 for the pump ratios in Fig. 10(a).

Figure 10(b) shows the log of the critical number of lasers as a function of the log of the coupling strength for different magnitudes of noise of a random phase field with relative intensity $I_{\xi}$ added to each star laser. The black dashed line corresponds to a power law $M_{c}=a K^{v}$ fit to all the data with $v_{I}=-2.2$. As evident, the critical number of lasers as function of the coupling behave similarly for all noise levels. Note that for very high noise levels, the transition to synchronization is becoming less sharp.
[1] J. Aldridge and E. K. Pye, Cell density dependence of oscillatory metabolism, Nature (London) 259, 670 (1976).

[2] S. De Monte, F. d'Ovidio, S. Dan $\varnothing$, and P. G. Sørensen, Dynamical quorum sensing: Population density encoded in cellular dynamics, Proc. Natl. Acad. Sci. USA 104, 18377 (2007).

[3] I. R. Konigsberg, Diffusion-mediated control of myoblast fusion, Dev. Biol. 26, 133 (1971).

[4] S. Dan $\varnothing$, P. G. Sørensen, and F. Hynne, Sustained oscillations in living cells, Nature (London) 402, 320 (1999).

[5] A. Camilli and B. L. Bassler, Bacterial small-molecule signaling pathways, Science 311, 1113 (2006).

[6] A. F. Taylor, M. R. Tinsley, F. Wang, Z. Huang, and K. Showalter, Dynamical quorum sensing and synchronization in large populations of chemical oscillators, Science 323, 614 (2009).

[7] R. Toth, A. F. Taylor, and M. R. Tinsley, Collective behavior of a population of chemically coupled oscillators, J. Phys. Chem. B 110, 10170 (2006).

[8] M. Tinsley, A. Taylor, Z. Huang, F. Wang, and K. Showalter, Dynamical quorum sensing and synchronization in collections of excitable and oscillatory catalytic particles, Phys. D (Amsterdam) 239, 785 (2010).

[9] P. Dallard, A. J. Fitzpatrick, A. Flint, S. Le Bourva, A. Low, R. M. Ridsdill Smith, and M. Willford, The London Millennium Footbridge, Struct. Engineer 79, 17 (2001).

[10] S. H. Strogatz, D. M. Abrams, A. McRobie, B. Eckhardt, and E. Ott, Crowd synchrony on the Millennium Bridge, Nature (London) 438, 43 (2005).
[11] B. Eckhardt, E. Ott, S. H. Strogatz, D. M. Abrams, and A. McRobie, Modeling walker synchronization on the Millennium Bridge, Phys. Rev. E 75, 021110 (2007).

[12] I. Belykh, R. Jeter, and V. Belykh, Foot force models of crowd dynamics on a wobbly bridge, Sci. Adv. 3, e1701512 (2017).

[13] E. Ingólfsson, C. Georgakis, F. Ricciardelli, and J. Jönsson, Experimental identification of pedestrian-induced lateral forces on footbridges, J. Sound Vib. 330, 1265 (2011).

[14] S. Živanović, A. Pavic, and P. Reynolds, Vibration serviceability of footbridges under human-induced excitation: a literature review, J. Sound Vib. 279, 1 (2005).

[15] V. Joshi and M. Srinivasan, Walking crowds on a shaky surface: stable walkers discover millennium bridge oscillations with and without pedestrian synchrony, Biol. Lett. 14, 20180564 (2018).

[16] J. Zamora-Munt, C. Masoller, J. Garcia-Ojalvo, and R. Roy, Crowd Synchrony and Quorum Sensing in Delay-Coupled Lasers, Phys. Rev. Lett. 105, 264101 (2010).

[17] E. Cohen, M. Rosenbluh, and I. Kanter, Phase transition in crowd synchrony of delay-coupled multilayer laser networks, Opt. Express 20, 19683 (2012).

[18] T. Gregor, K. Fujimoto, N. Masaki, and S. Sawai, The onset of collective behavior in social amoebae, Science 328, 1021 (2010).

[19] D. Helbing, D. Brockmann, T. Chadefaux, K. Donnay, U. Blanke, O. Woolley-Meza, M. Moussaid, A. Johansson, J. Krause, S. Schutte, and M. Perc, Saving human lives: What complexity science and information systems can contribute, J. Stat. Phys. 158, 735 (2015). 
[20] T. Danino, O. Mondragón-Palomino, L. Tsimring, and J. Hasty, A synchronized quorum of genetic clocks, Nature (London) 463, 326 (2010)

[21] J. Garcia-Ojalvo, M. B. Elowitz, and S. H. Strogatz, Modeling a synthetic multicellular clock: Repressilators coupled by quorum sensing, Proc. Natl. Acad. Sci. USA 101, 10955 (2004).

[22] J. Gómez-Gardeñes, S. Gómez, A. Arenas, and Y. Moreno, Explosive Synchronization Transitions in Scale-Free Networks, Phys. Rev. Lett. 106, 128701 (2011).

[23] I. Leyva, R. Sevilla-Escoboza, J. M. Buldú, I. Sendina-Nadal, J. Gómez-Gardeñes, A. Arenas, Y. Moreno, S. Gómez, R. JaimesReátegui, and S. Boccaletti, Explosive First-Order Transition to Synchrony in Networked Chaotic Oscillators, Phys. Rev. Lett. 108, 168702 (2012)

[24] H. Singh and P. Parmananda, Crowd synchrony in chaotic oscillators, Nonlinear Dyn. 80, 767 (2015).

[25] L. V. Gambuzza, M. Frasca, and J. Gómez-Gardeñes, Intralayer synchronization in multiplex networks, Europhys. Lett. 110, 20010 (2015).

[26] E. Martz and M. S. Steinberg, The role of cell-cell contact in contact inhibition of cell division: A review and new evidence, J. Cell. Physiol. 79, 189 (1972).

[27] N. G. Berloff, M. Silva, K. Kalinin, A. Askitopoulos, J. D. Töpfer, P. Cilibrizzi, W. Langbein, and P. G. Lagoudakis, Realizing the classical XY Hamiltonian in polariton simulators, Nat. Mater. 16, 1120 (2017).

[28] M. Nixon, E. Ronen, A. A. Friesem, and N. Davidson, Observing Geometric Frustration with Thousands of Coupled Lasers, Phys. Rev. Lett. 110, 184102 (2013).

[29] P. L. McMahon, A. Marandi, Y. Haribara, R. Hamerly, C. Langrock, S. Tamate, T. Inagaki, H. Takesue, S. Utsunomiya, K. Aihara, R. L. Byer, M. M. Fejer, H. Mabuchi, and Y. Yamamoto, A fully programmable 100-spin coherent Ising machine with all-to-all connections, Science 354, 614 (2016).

[30] S. Mahler, M. L. Goh, C. Tradonsky, A. A. Friesem, and N. Davidson, Improved Phase Locking of Laser Arrays with Nonlinear Coupling, Phys. Rev. Lett. 124, 133901 (2020).

[31] V. Pal, S. Mahler, C. Tradonsky, A. A. Friesem, and N. Davidson, Rapid fair sampling of the XY spin Hamiltonian with a laser simulator, Phys. Rev. Research 2, 033008 (2020).

[32] A. Marandi, Z. Wang, K. Takata, R. L. Byer, and Y. Yamamoto, Network of time-multiplexed optical parametric oscillators as a coherent ising machine, Nat. Photonics 8, 937 (2014).

[33] V. Pal, C. Tradonsky, R. Chriki, A. A. Friesem, and N. Davidson, Observing Dissipative Topological Defects with Coupled Lasers, Phys. Rev. Lett. 119, 013902 (2017).

[34] C. Tradonsky, I. Gershenzon, V. Pal, R. Chriki, A. A. Friesem, O. Raz, and N. Davidson, Rapid laser solver for the phase retrieval problem, Sci. Adv. 5, eaax4530 (2019).
[35] M. C. Soriano, J. García-Ojalvo, C. R. Mirasso, and I. Fischer, Complex photonics: Dynamics and applications of delaycoupled semiconductors lasers, Rev. Mod. Phys. 85, 421 (2013).

[36] M. Komarov and A. Pikovsky, Finite-size-induced transitions to synchrony in oscillator ensembles with nonlinear global coupling, Phys. Rev. E 92, 020901(R) (2015).

[37] A. Pikovsky, A. Zaikin, and M. A. de la Casa, System Size Resonance in Coupled Noisy Systems and in the Ising Model, Phys. Rev. Lett. 88, 050601 (2002).

[38] S. Acharyya and R. E. Amritkar, Synchronization of nearly identical dynamical systems: Size instability, Phys. Rev. E 92 052902 (2015).

[39] N. Nair, E. Bochove, and Y. Braiman, Almost perfect in-phase and anti-phase chaotic and periodic phase synchronization in large arrays of diode lasers, Opt. Commun. 430, 104 (2019).

[40] S. Y. Kourtchatov, V. V. Likhanskii, A. P. Napartovich, F. T. Arecchi, and A. Lapucci, Theory of phase locking of globally coupled laser arrays, Phys. Rev. A 52, 4089 (1995).

[41] J. A. Arnaud, Degenerate optical cavities, Appl. Opt. 8, 189 (1969).

[42] M. Nixon, M. Friedman, E. Ronen, A. A. Friesem, N. Davidson, and I. Kanter, Synchronized Cluster Formation in Coupled Laser Networks, Phys. Rev. Lett. 106, 223901 (2011).

[43] C. Tradonsky, V. Pal, R. Chriki, N. Davidson, and A. A. Friesem, Talbot diffraction and Fourier filtering for phase locking an array of lasers, Appl. Opt. 56, A126 (2017).

[44] S. Mahler, C. Tradonsky, R. Chriki, A. A. Friesem, and N. Davidson, Coupling of laser arrays with intracavity elements in the far-field, OSA Continuum 2, 2077 (2019).

[45] M. Nixon, O. Katz, E. Small, Y. Bromberg, A. A. Friesem, Y. Silberberg, and N. Davidson, Real-time wavefront shaping through scattering media by all-optical feedback, Nat. Photonics 7, 919 (2013).

[46] A. G. Fox and T. Li, Resonant modes in a maser interferometer, Bell Syst. Tech. J. 40, 453 (1961).

[47] R. W. Gerchberg and W. O. Saxton, A practical algorithm for the determination of the phase from image and diffraction plane pictures, Optik 35, 237 (1972).

[48] R. A. Oliva and S. H. Strogatz, Dynamics of a large array of globally coupled lasers with distributed frequencies, Int. J. Bifurcation Chaos 11, 2359 (2001).

[49] J. Zamora-Munt and E. Ott, Double transition to synchronization: A generic emergent transitional behavior in large systems of coupled oscillators, Europhys. Lett. 98, 40007 (2012).

[50] R. Chriki, S. Mahler, C. Tradonsky, V. Pal, A. A. Friesem, and N. Davidson, Spatiotemporal supermodes: Rapid reduction of spatial coherence in highly multimode lasers, Phys. Rev. A 98, 023812 (2018).

[51] D. Witthaut and M. Timme, Kuramoto dynamics in Hamiltonian systems, Phys. Rev. E 90, 032917 (2014). 